\section{Auswirkung des Fastens im Ramadan auf den Fetus}

\author{
Entgegen religiöser Vorschriften nehmen schwangere Muslime im Ramadan \\ oft am Fasten teil. Welche Auswirkungen die verminderte Nahrungsaufnahme \\ möglicherweise auf den Nachkommen - auch im späteren Leben - hat, \\ wurde nun in einer Studie untersucht.
}

$\mathrm{M}$ ehrere Stuiden der letzten Jahre belegen einen Zusammenhang zwischen einer mütterlichen Diät während der Schwangerschaft und deren Effekt auf die Gesundheit des Kindes im weiteren Leben. Es wurde gezeigt, dass Kinder, deren Mütter während der Schwangerschaft die Nahrungsaufnahme erheblich reduzierten, einen kleineren BodyMass-Index (BMI) hatten. Unklar ist, ob ein intermittierendes Fasten während des muslimischen Fastenmonats Ramadan auch eine Auswirkung auf den zukünftigen BMI des Kindes hat. Während des Ramadans dürfen gläubige Muslime von Sonnenauf- bis Sonnenuntergang weder Flüssigkeit noch Nahrung zu sich nehmen. Schwangere sind von dieser religiösen Vorschrift ausgenommen. Al- lerdings nehmen $70-90 \%$ der schwangeren Muslime aus familiären oder gesellschaftlichen Gründen an diesem Brauch teil. Den Einfluss des Fastens während des Ramadans auf die Schwangerschaft, untersuchte nun eine populationsbezogene Studie in Indonesien. Bei einer Auswertung der Daten des indonesischen Family Life Survey aus dem Jahr 2000 wurde der BMI von Muslimen, die während des Ramadans in utero waren, mit denen verglichen, deren Schwangerschaft nicht in den Ramadan fiel. Die Studienpopulation umfasste 14.120 Muslime und 1.220 Nicht-Muslime mit einem Durchschnittsalter von 34,6 Jahren. Erwachsene Muslime, die während des Ramadans in utero waren, waren geringfügig dünner als die Vergleichsgrup- pe (BMI: -0,32, 95\% Konfidenzinervall: $-0,57 ;-0,06)$. Erfolgte die Befruchtung während des Ramadans ergab sich im späteren Leben eine um $0,8 \mathrm{~cm}$ geringere Körpergröße im Vergleich mit denjenigen ohne Bezug zum Ramadan. Die Vergleichsgruppe der Nicht-Muslime wies hingegen keine Auffälligkeiten hinsichtlich der Körpergröße und des BMI auf. Die Autoren folgern daher, dass auch das intermittierende Fasten im Ramadan während der Schwangerschaft eine Auswirkung auf die Entwicklung des Fetus hat.

Fazit: Als Ursache für das im späteren Leben beobachtete geringere Längenwachstum wird Fasten-bedingt eine Reduktion der Plazentagröße vermutet. Weil es hierzulande viele weibliche Muslime gibt, hat die Studie auch für Deutschland Relevanz. Prof. Tino F. Schwarz

Van Ewijk RJG et al.. Associations of prenatal exposure to Ramadan with small stature and thinness in adulthood: Results from a large Indonesian population-based study. Am J Epidemiol 2013; 177:729-36

\title{
Beckenbodenchirurgie: mehr Harnwegsinfekte bei positiver Urinkultur am OP-Tag
}

Trotz perioperativer Antibiotikatherapie ist bei Frauen nach einer Beckenbodenchirurgie mit positiver Urinkultur am Tag des Eingriffs die Rate der Harnwegsinfektionen deutlich erhöht. In einer kleinen Studie aus den USA war der Anteil positiver Kulturen am Tag der Operation viel höher als erwartet.

7 wei wei große US-amerikanische Studien brachten zutage, dass nach einer Beckenbodenchirurgie wegen eines Prolapses oder einer Harninkontinenz die Rate postoperativer Harnwegsinfektionen (HWI) zwischen 7\% und 24\% liegt. Und sogar bei fast jeder zweiten älteren Frau, bei der eine obliterative Beckenbodenchirurgie vorgenommen wurde, kommt es postoperativ zu solchen Infektionen. Das Problem könnte sich verschärfen, wenn die Prognose zutrifft, dass im Jahr 2050 weltweit mehr als 58 Millionen Frauen Erkrankungen des Beckenbodens haben.

In einer Studie mit 284 Teilnehmerinnen prüften Urogynäkologen, ob eine positive Urinkultur am Tag der

Operation ein Risikofaktor für spätere HWI ist. Als positiv wurde ein Testergebnis gewertet, wenn eine Kultur mindestens 1.000 Bakterienkolonien pro Milliliter enthielt. In der prospektiven Studie waren insgesamt 9,4\% $(\mathrm{n}=27)$ der am Tag der Operation vorgenommenen Urinkulturen positiv. Sie enthielten typische Uropathogene wie Escherichia coli, Pseudomonaden, Klebsiellen und Staphylokokken, aber auch Corynebakterien, Streptococcus agalactiae und Laktobazillen. Zehn Patientinnen mit positivem Test wurden postoperativ antibiotisch behandelt. Bei keiner Patientin kam es jedoch später zu Komplikationen der Infektion (z.B. Pyelonephritis oder Urosepsis). Im
Vergleich zu Patientinnen mit negativer Urinkultur war bei Patientinnen mit positivem Test der Anteil der Frauen stark erhöht, die trotz standardmäßig perioperativ verabreichter Antibiotika nach dem Eingriff HWI entwickelten $(29,6 \%$ vs. $5,6 \% ; p=0,005)$.

Fazit: Gelänge es, jene Frauen zu identifizieren, bei denen die Wahrscheinlichkeit für positive Urinkulturen am Tag des Eingriffs deutlich erhöht ist, könnten postoperative HWI leichter abgewehrt werden, so die Autoren. Leider hätten sie solche Faktoren in ihrer kleinen Studie nicht ausmachen können. Um HWI zu verhindern, reicht zudem möglicherweise eine einmalige intravenös verabreichte Antibiotika-Applikation nicht aus, wie frühere Studien vermuten lassen.

Peter Leiner

Fok CS et al. Day of Surgery Urine Cultures Identify Urogynecologic Patients at Increased Risk for Post-operative Urinary Tract Infection. J Urol 2013; 189: 1721-4 